University of Nebraska - Lincoln

DigitalCommons@University of Nebraska - Lincoln

$12-28-2002$

\title{
Perturbative calculation of the triply differential cross section for photo-double-ionization of $\mathrm{He}$
}

Andrei Y. Istomin

University of Nebraska - Lincoln

N. L. Manakov

Voronezh State University, manakov@phys.vsu.ru

Anthony F. Starace

University of Nebraska-Lincoln, astarace1@unl.edu

Follow this and additional works at: https://digitalcommons.unl.edu/physicsstarace

Part of the Physics Commons

Istomin, Andrei Y.; Manakov, N. L.; and Starace, Anthony F., "Perturbative calculation of the triply differential cross section for photo-double-ionization of He" (2002). Anthony F. Starace Publications. 137. https://digitalcommons.unl.edu/physicsstarace/137

This Article is brought to you for free and open access by the Research Papers in Physics and Astronomy at DigitalCommons@University of Nebraska - Lincoln. It has been accepted for inclusion in Anthony F. Starace Publications by an authorized administrator of DigitalCommons@University of Nebraska - Lincoln. 
Published in Journal of Physics B: Atomic, Molecular and Optical Physics 35:24 (December 28, 2002), pp. L543-L552. Copyright (C) 2002 IOP Publishing Ltd. Used by permission.

http://stacks.iop.org/JPhysB/35/L543

Submitted September 22, 2002; revised November 17, 2002; published online December 4, 2002.

LETTER TO THE EDITOR

\title{
Perturbative calculation of the triply differential cross section for photo-double-ionization of He
}

\author{
Andrei Y. Istomin ${ }^{1}$, N. L. Manakov ${ }^{2}$, and Anthony F. Starace ${ }^{1}$ \\ ${ }^{1}$ Department of Physics and Astronomy, University of Nebraska-Lincoln, Lincoln, \\ NE 68588-0111, USA \\ ${ }^{2}$ Research and Education Center, Voronezh State University, Voronezh 394006, \\ Russia
}

\begin{abstract}
Single-photon, two-electron ionization of He is analyzed, taking into account electron correlation using lowest-order perturbation theory and including all individual electron angular momenta in the final two-electron continuum. Perturbative account of electron correlation in the final state, which describes the so-called TS- 1 mechanism of double photoionization, combined with a variational account of electron screening, is found to provide results for the triply differential cross section at an excess energy of $20 \mathrm{eV}$ that are in excellent agreement with both absolute experimental data and results of non-perturbative calculations, for all kinematics of the process in which the TS-1 mechanism is expected to dominate.
\end{abstract}

For many decades, the process of single-photon, double ionization-or photo-doubleionization (PDI) - of the He atom has been of intense interest, as a probe of electron correlations $[1,2]$. Owing to the difficulty of describing the double-continuum final state (FS), most theoretical treatments have employed significant approximations. Initially, theorists used correlated ground state (GS) wavefunctions and uncorrelated FS wavefunctions calculated in the field of the doubly charged $(Z=2)$ He nucleus [3]. By the mid1970s, however, theorists (e.g., [4-8]) turned to perturbation theory (PT) to treat correlation effects between the two ionized electrons. In the past decade or so, attention has shifted from the total PDI cross section to the triply differential cross section (TDCS), which describes the angular distribution of the two ionized electrons and which is a much more sensitive test of theoretical approximations and models. Theoretical treatments initially reverted to using correlated GS wavefunctions and different kinds of improved (often analytical) FS wavefunction (see the review [1]). In general, even though the TDCS angular patterns were reproduced qualitatively, in most works where comparisons with absolute experimental data were made (see, e.g., [9]), various scaling factors had to be introduced, although there have been exceptions [10]. The recent availability of absolute experimental data for the TDCS (see reviews [1, 2]) has stimulated non-perturbative numerically intensive treatments for the TDCS [12-16]. Results of these ab initio treatments are generally gauge invariant and in excellent agreement with experimental 
measurements, and therefore provide a benchmark for testing simpler theoretical approaches, whose main value lies in elucidating the electron correlation mechanisms leading to PDI. The purpose of this letter is to examine one such approximate approach, the lowest-order perturbative analysis of electron correlations in PDI.

From these nearly four decades of theoretical analyses, we note here several aspects that are relevant to our perturbative analysis. First, approximate treatments of the PDI TDCS are highly sensitive to the gauge in which the electric dipole interaction is evaluated $[17,18]$; for low photon energies, the velocity gauge is least sensitive to higher-order perturbative corrections [5] and gives the best absolute values for the TDCS [18]. Second, the TDCS is very sensitive to final state correlations (FSC) in all gauges, while for the case of equal energy sharing it is insensitive to the precise form of the GS wavefunction [18]. (Indeed, the total double-ionization cross section for low photon energies $(\leq 500$ $\mathrm{eV}$ ) may be described quite accurately (in the velocity gauge) by taking only FSC into account [7].) Third, the detailed PT analyses in [5] and [8] provide much information on high-order correlation terms. Specifically, many higher-order FSC terms having to do with electron screening effects can be accounted for by using two different basis sets for the ionized electrons, with the faster electron seeing a net charge of $Z=1$ and the slower electron seeing a net charge of $Z=2$. Regarding ground state correlation (GSC), higherorder terms tend to cancel the lowest-order GSC amplitude [5].

Despite the long history of theoretical analyses of the PDI process in He, surprisingly the first actual lowest-order perturbation theory (LOPT) calculation of the TDCS was reported only recently [11] (for an excess energy of $20 \mathrm{eV}$ ). A basis of $Z=2$ Coulomb functions was employed, the experimental (rather than theoretical) double-ionization energy was used in the PT energy denominators and the FS partial wave series was truncated. For both symmetric and asymmetric energy sharings, the theoretical results [11] must be scaled by factors ranging from 0.10 to 0.19 in order to be compared with the absolute experimental data [9]. Within the velocity gauge, which is the only one used in [11], GSC is predicted to have only a small influence on the TDCS relative to FSC.

In this letter we revisit the perturbative evaluation of the TDCS for PDI of He, using a new calculational technique. We present two different sets of calculations for the case where the ionized electrons share an excess energy of $E_{1}+E_{2}=20 \mathrm{eV}=\omega+E_{0}$, where $E_{1}$ and $E_{2}$ are the photoelectron energies, $\omega$ is the photon energy and $E_{0}$ is the GS energy of He. First, we present LOPT results with a basis of $Z=2$ Coulomb wavefunctions calculated in the length $(L)$ and velocity $(V)$ gauges. For this case, we present two versions, corresponding to the two common ways of handling $\omega+E_{0}=20 \mathrm{eV}$, i.e. using either experimental $\left(E_{0}^{\text {exp }}\right)$ or theoretical $\left(E_{0}^{\text {th }}\right)$ values for $E_{0}$. Second, we present results of a simple set of approximations: the GS is described by a pair of independent-particle bound-state Coulomb wavefunctions having the variationally determined effective charge $Z=27 / 16$; the FS is described by $Z=27 / 16$ Coulomb continuum wavefunctions and FS electron correlation is taken into account to first order. We show that this simple approach provides excellent quantitative agreement with both absolute experimental data [9] and results of recent non-perturbative theoretical calculations [9, 13-15].

The dipole transition matrix element for PDI (in the $V$ gauge) is (in atomic units)

$$
M\left(\boldsymbol{e}, \boldsymbol{p}_{1}, \boldsymbol{p}_{2}\right)=\left\langle\psi_{p_{1} p_{2}}\left(\boldsymbol{r}_{1}, \boldsymbol{r}_{2}\right)\left|\boldsymbol{e} \cdot\left(\nabla_{1}+\nabla_{2}\right)\right| \psi_{E_{0}}\left(\boldsymbol{r}_{1}, \boldsymbol{r}_{2}\right)\right\rangle,
$$

where $p_{1}$ and $p_{2}$ are the FS photoelectron momenta, and where $e$ is the unit (in general, complex) photon polarization vector, $e \cdot e^{*}=1$. Provided the FS wavefunction is momen- 


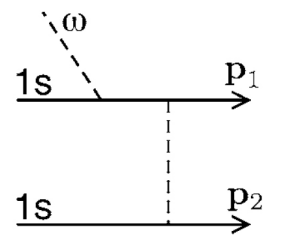

(a)

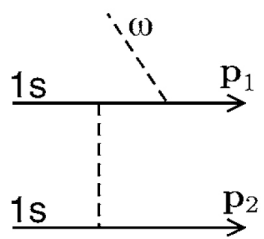

(b)

Figure 1. Schematic diagrams contributing to the PDI process in the first order of PT. (a) FSC, final state correlations; (b) GSC, ground state correlation. Two additional diagrams with exchanged $p_{1}$ and $p_{2}$ are included.

tum normalized, the TDCS is given by (where $\alpha=1 / 137.036$ )

$$
\frac{\mathrm{d}^{3} \sigma}{\mathrm{d} \Omega_{1} \mathrm{~d} \Omega_{2} \mathrm{~d} E_{1}}=\frac{4 \pi^{2} \alpha}{\omega} p_{1} p_{2}\left|M\left(e, p_{1}, p_{2}\right)\right|^{2} .
$$

To evaluate $M$, we approximate in our first set of calculations the exact initial and FS wavefunctions, $\psi_{E 0}$ and $\psi_{p 1 p 2}$ by taking into account the first-order corrections (in $1 / r_{12}$ ) to the unperturbed basis states, for which we use symmetrized products of independentparticle Coulomb orbitals with charge $Z=2$. Thus, $M$ is given by a sum of four amplitudes, which are presented schematically in Figure 1. The FSC diagram describes the process in which one electron absorbs the photon and then interacts with the other electron, exciting it to a continuum state. The GSC diagram describes the process in which the GS electrons interact and are virtually excited, whereupon the photon is absorbed by one of the electrons, providing enough energy for both electrons to be ionized. These diagrams can be related to those appearing in the many-body PT analysis of electron correlations (see, e.g., [7]). Specifically, the FSC amplitude in Figure 1(a) is given identically by equation (11) of [7], which those authors call the TS-1 amplitude. Our GSC amplitude in Figure 1(b) is given identically by equation (13) of [7] provided one retains the term involving $k=1 s$ in that equation (and uses the relations $\omega=E_{1}+E_{2}-E_{0}$ and $E_{0}=2 \epsilon_{1 s}$ ), i.e., it includes both the "GSC" and the "SO" (shake-off) terms of [7].

A detailed description of our computational technique, which allows one to account for all individual electron angular momenta in the final two-electron Coulomb continuum state, will be presented elsewhere [19]. In brief, it uses the integral representation for the electron correlation operator

$$
\frac{1}{r_{i j}}=\frac{1}{2 \pi^{2}} \int \mathrm{d} q \frac{\mathrm{e}^{\mathrm{i} q \cdot r_{i j}}}{q^{2}}
$$

and the integral representation for the Coulomb Green function (CGF), $G_{\mathcal{E}}\left(r, r^{\prime}\right)$, in parabolic coordinates [20], $\xi=r+z, \eta=r-z, \varphi=\arctan (y / x)$ :

$$
\begin{gathered}
G_{\mathcal{E}}\left(\xi, \eta, \varphi ; \xi^{\prime}, \eta^{\prime}, \varphi^{\prime}\right)=\frac{1}{\pi v} \int_{0}^{1} \mathrm{~d} \tau \frac{\tau^{-Z v}}{(1-\tau)^{2}} \exp \left(-\frac{\xi+\xi^{\prime}+\eta+\eta^{\prime}}{2 v} \frac{1+\tau}{1-\tau}\right) \\
\times \sum_{m} I_{m}\left(\frac{2 \sqrt{\tau \xi \xi^{\prime}}}{v(1-\tau)}\right) I_{m}\left(\frac{2 \sqrt{\tau \eta \eta^{\prime}}}{v(1-\tau)}\right) \mathrm{e}^{\mathrm{i} m\left(\varphi-\varphi^{\prime}\right)},
\end{gathered}
$$

where $v=1 /(-2 \mathcal{E})^{1 / 2}$ and $I_{m}(z)$ is the modified Bessel function. With these representations and using known results for the Coulomb continuum ingoing waves in parabolic coordinates (using the one-dimensional integral representation for one of them), all three spatial (three-dimensional) integrals in the first-order perturbation matrix elements corre- 
sponding to the diagrams in Figure 1 are evaluated analytically, in terms of algebraic functions of the variable $\tau$ in (4) and components of the vector $q$ in (3). (Note that owing to dipole selection rules, only the terms with $m=0, \pm 1$ in (4) contribute in our case.) Thus, finally, we have four integrations: over $\tau$ and $q$. Since the integral over the azimuthal angle of $\boldsymbol{q}$ is independent of the integration over $\tau$, there remain only three-dimensional integrals to be evaluated numerically.

In any perturbative treatment of an atomic photoionization process, one must account for electron correlation effects, not only on the transition matrix elements but also on the various energies involved. For the double-ionization process, however, the transition amplitudes are identically zero, unless electron correlation is taken into account. Hence, in a consistent PT treatment of electron correlation to first order, the zero-order theoretical energies must be used, in order to maintain gauge invariance. The problem is that the zero-order energies may be rather different from the experimental ones. A common response is to insert experimental values for the various energies involved and to focus the theoretical effort on the dynamical matrix elements, rather than on the stationary state energies. If one does this, however, the results become gauge dependent. In our first set of calculations for the TDCS, we investigate both approaches to LOPT using a basis of $Z=2$ Coulomb functions:

(i) the use of the theoretical energies $E_{0}{ }^{\text {th }}=-Z^{2}$ and $\epsilon_{1 s}=-Z^{2} / 2$ (which, if one fixes the excess energy $E_{1}+E_{2}=20 \mathrm{eV}=\omega+E_{0}^{\text {th }}$, requires that $\omega$ is shifted from its experimental value by $29.64 \mathrm{eV})$;

(ii) the use of the experimental value for $E_{0}(-79.2 \mathrm{eV})$ and setting $\epsilon_{1 s}=E_{0} / 2$, which is the approach used by Keller [11].

In case (i) the total transition amplitude, $M=M_{G S C}+M_{F S C}$ (as well as the TDCS), is gauge invariant (although the separate FSC and GSC amplitudes are gauge dependent), while in case (ii) the gauge invariance of $M$ is lost [19].

The results for the TDCS in these two possible $Z=2$ LOPT versions are compared with absolute experimental data [9] for the case of linearly polarized photons at an excess energy of $20 \mathrm{eV}$ in Figure 2. Our results are shown for both the $L$ and $V$ gauges and include an exact account of all individual electron angular momenta. (In the figures, $\mathrm{F}$ and G stand for FSC and GSC.) As they should be, the TDCSs in the first, gauge-independent LOPT version (using $E_{0}^{\text {th }}$ ) are identical in the $L$ and $V$ gauges (see the $G+F$ curves in bold in Figures 2(a) and (b)), even though the separate GSC and FSC contributions are significantly gauge dependent. In the $E_{0}^{\text {exp }}$-version (cf. Figures $2(\mathrm{c})-(\mathrm{f})$ ), the total TDCSs in the $L$ and $V$ gauges, while not identical, are close in both shape and magnitude. They differ in magnitude, however, by factors of four to six from those in Figures 2(a) and (b). In both LOPT versions, the TDCSs in which only GSC is taken into account, TDCS(GSC), are of the same order of magnitude or even larger than the corresponding TDCS(FSC), in which only FSC is taken into account. Our $V$-gauge results in the LOPT version using $E_{0}^{e x p}$ (Figures 2(c) and (d)) differ from the predictions in Figures 3(a) and (c) of [11] in both shape and magnitude. In particular, our FV results for the TDCS are close to the experimental ones in both shape and magnitude without any scaling. In contrast to [11], our GV results for the TDCS are a few times larger than our FV results. Although we have endeavored to ensure that our second LOPT set of calculations (in Figures 2(c), (d)) corresponds exactly to that of [11], we are unable to account for the differences just mentioned.

To confirm the results of our new technique, we have carried out independent calculations using the conventional partial wave expansions of the CGF in spherical coordinates, taking into account only $l_{1}, l_{2}=0,1,2$ partial waves in the one-electron continuum states. The results (using $E_{0}^{\text {th }}$ ) are given in Figure 3 for the $V$-gauge and are compared 

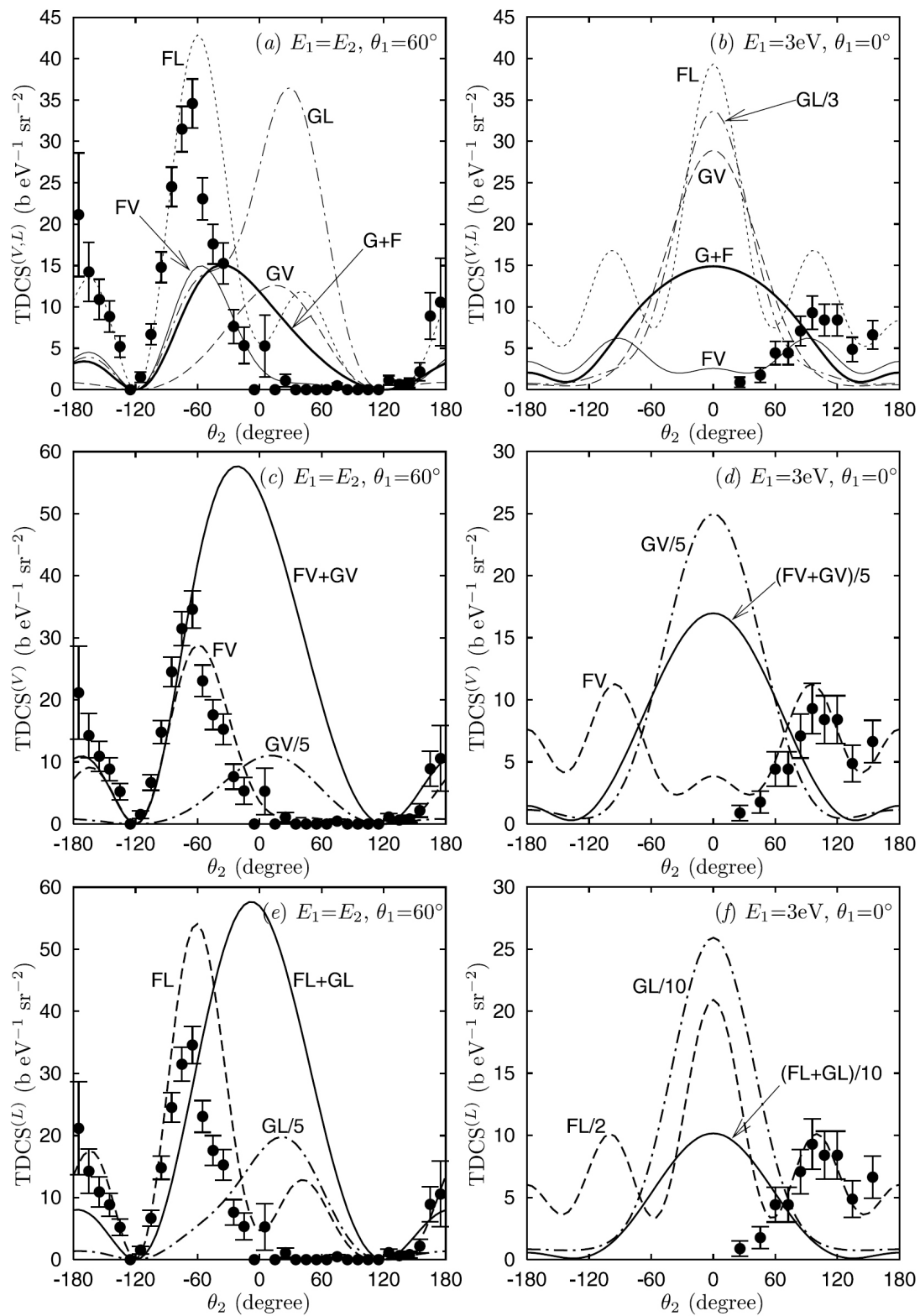

Figure 2. LOPT results for the TDCS in the $L$ and $V$ gauges using a basis of $Z=2$ Coulomb oneparticle orbitals with an exact account of all individual electron angular momenta. The excess energy is $20 \mathrm{eV}$, coplanar geometry is assumed and the photon polarization is along $\theta=0^{\circ}$. (a), (b) Gauge-independent LOPT results ( $V$ and $L$ gauges) using $E_{0}^{\text {th }}$; (c), (d) $V$-gauge results using $E_{0}^{\text {exp }} ;$ (e), (f) $L$-gauge results using $E_{0}^{\text {exp }}$. The notations $G$ and $F$ stand for account of GSC and FSC respectively. Full bold curve in $(a),(b)=$ identical result for the $\operatorname{TDCS}(G+F)$ in $L$ and $V$ gauges. Absolute experimental data from Bräuning et al. [9].

with the corresponding results for our first LOPT version in Figures 2(a) and (b). By comparing the results of these two calculations, we are able both to check our exact calculation for accuracy and also to estimate the contribution of higher individual electron angular momenta. One sees that taking into account only s, p and d waves gives an accurate prediction of the angular dependence of TDCS(FSC) and TDCS(GSC) for a wide 

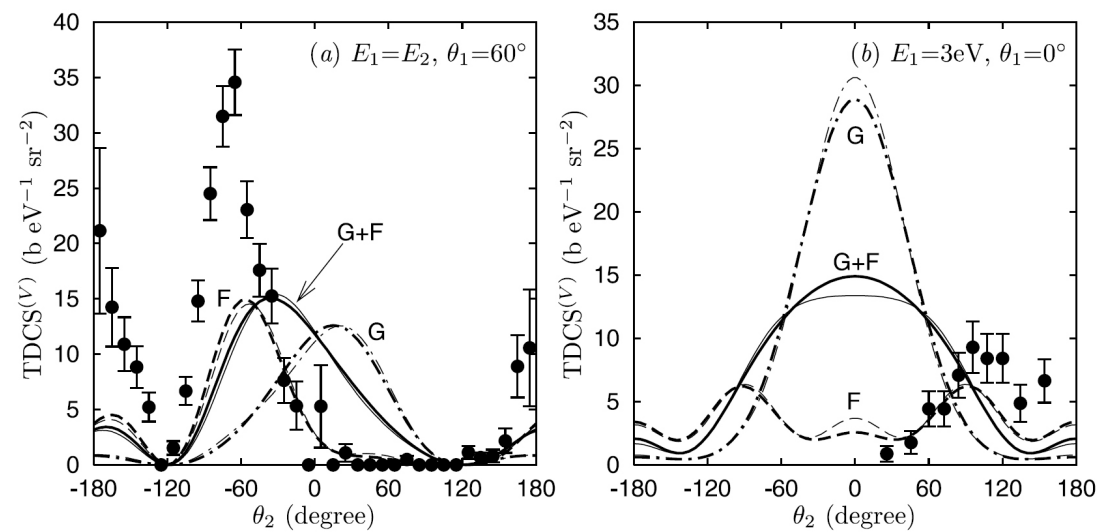

Figure 3. Comparison of LOPT results (using $Z=2, E_{0}^{\text {th }}$ ) with exact account of all individual electron angular momenta (bold curves) to the corresponding results with account of only $\mathrm{s}, \mathrm{p}$ and d waves (curves of regular thickness). Full curves = FSC + GSC; dashed curves = FSC only; dot-dashed curves = GSC only. Absolute experimental data from Bräuning et al. [9].

range of mutual angles $\theta_{12}$, although significant effects of higher angular momenta are seen at small $\theta_{12}$ (cf. Figure $\left.3(\mathrm{~b})\right)^{*}$. Note, finally, that partial wave expansion results using $E_{0}^{e x p}$ agree similarly well with the second LOPT version results in Figures 2(c) and (d); thus, the truncation of partial wave series in [11] cannot explain the discrepancies between our results in Figures 2(c) and (d) and those in [11].

As one sees from Figure 2, in both gauges and in both LOPT versions, the FSC amplitude predicts an angular dependence of the TDCS that is qualitatively similar to that of the experimental data both in shape and magnitude, while the GSC amplitude gives a TDCS having a very different angular distribution: for $E_{1}=3 \mathrm{eV}, \theta_{1}=0$, it predicts a maximum at $\theta_{12}=0$, while for $E_{1}=E_{2}$ and $\theta_{1}=60^{\circ}$, it predicts a peak closer to $\theta_{12}$ $=0$ than experiment does. Thus, the GSC amplitude dramatically affects the shape of TDCS(FSC + GSC). That this effect is exaggerated in first-order PT can be understood by a detailed analysis of higher-order terms of the PT series [5]: in higher orders of the PT series, the diagrams of the same class as the first-order GSC hole-hole interaction diagram (i.e. those which are calculated with an uncorrelated FS) exhibit significant cancellations with the lowest-order GSC diagram. Account of the GSC diagram only in the first order is thus inadequate; it obviously influences the predicted angular dependence of the TDCS(FSC + GSC) more than it should, nevertheless serving to make it gauge invariant in the LOPT version in which $E_{0}^{\text {th }}$ is used. As shown in [5], reduction of the effects of GSC in first order requires essentially an infinite summation of higher-order diagrams. Carter and Kelly [5] carried out this summation approximately, estimating that the lowest-order GSC amplitude was reduced by a factor of 0.768 for the $k s k p$ channel and 0.890 for the $k p k d$ channel. Additional reductions of the GSC terms were found due to higher-order energy shifts. Our conclusion, based on the detailed PT analyses in $[5,8]$ and on our results, is that FSC can be taken into account to first order, while GSC cannot. Indeed, as our LOPT calculations of the TDCS in two different versions show, the first-order GSC matrix elements dramatically affect the shape of the TDCS, in a way that disagrees both with predictions using only FSC and with experimental measurements.

We report here a second set of calculations, based on a simple set of approximations. Owing to the difficulty of describing GSC effects perturbatively, we instead ap-

* Similar differences are found (but not shown) for $\theta_{1}=\theta_{2}=0$ in the equal energy-sharing case. 

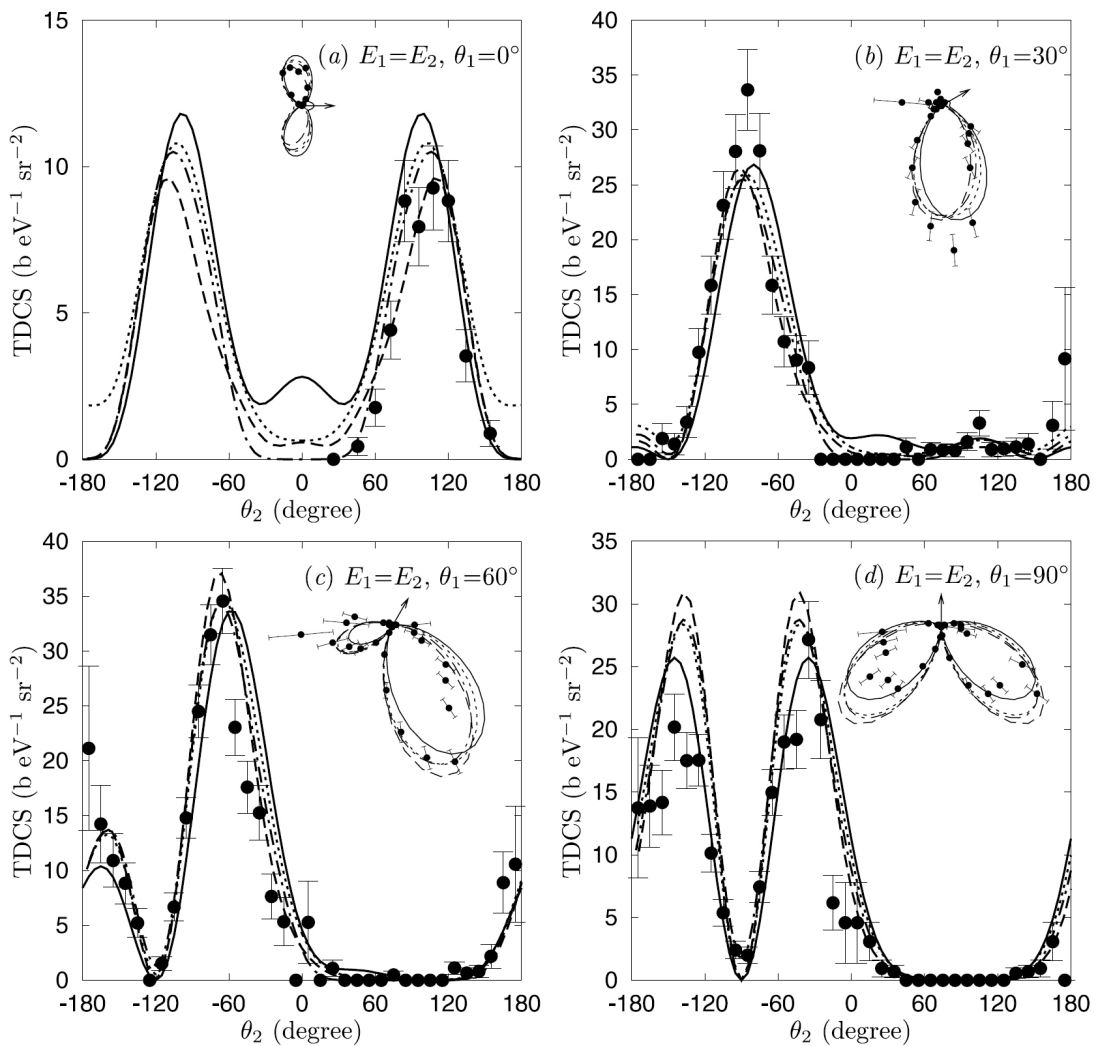

Figure 4. TDCS for PDI of He at an excess energy of $20 \mathrm{eV}$ for equal energy sharing for various angles of ejection $\theta_{1}$. The polar plots for the TDCS in (a)-(d) are all given on the same scale; the arrow shows the direction of emission of the electron having energy $E_{1}$, and the photon polarization is directed to the right. Full curves $=$ present $V$ gauge results using a basis of Coulomb one-particle orbitals calculated for $Z=27 / 16$ and using perturbative account of FSC to lowest order; dashed curves $=$ TDCC results of Colgan et al. [15]; dotted curves $=$ CCC results of Kheifets and Bray [9]; dot-dashed curves= HRM-SOW results of Selles et al. [14]. Absolute experimental data from Bräuning et al. [9].

proximate the He GS by a pair of Coulomb orbitals, $\psi_{E 0}=\left(Z^{3} / \pi\right) \exp \left[-Z\left(r_{1}+r_{2}\right)\right]$, with the variationally determined charge, $Z=27 / 16$. (The same description of the GS wavefunction was used recently in evaluating the TS-1 amplitude quasi-classically [21].) For consistency, we use $Z=27 / 16$ in the intermediate (CGF) and final one-electron continuum states. We treat FSC effects to first order in the electron-correlation operator and employ the $V$ gauge, as this one is the least sensitive to higher-order correlation effects [5]. Finally, since the variational double ionization potential, $\left|E_{0}^{\text {th }}\right| \approx 77.5 \mathrm{eV}$, is very close to $\mid$ $E_{0}^{\exp } \mid=79.2 \mathrm{eV}$, the choice for $E_{0}$ is of far less significance than for the LOPT calculations with $Z=2$ presented above; we use here $E_{0}^{\text {th }}$.

In Figures 4-6 we present the results of our second set of calculations of the TDCS using the approach described above, for the case of an excess energy of $20 \mathrm{eV}$ for four different ejection angles of one electron and for both equal and unequal energy sharing. All individual electron angular momenta are taken into account. We compare our results with the absolute experimental data of [9], with the convergent close-coupling (CCC) results of Kheifets and Bray $[9,13]$, with the hyperspherical $R$-matrix with semiclassi- 

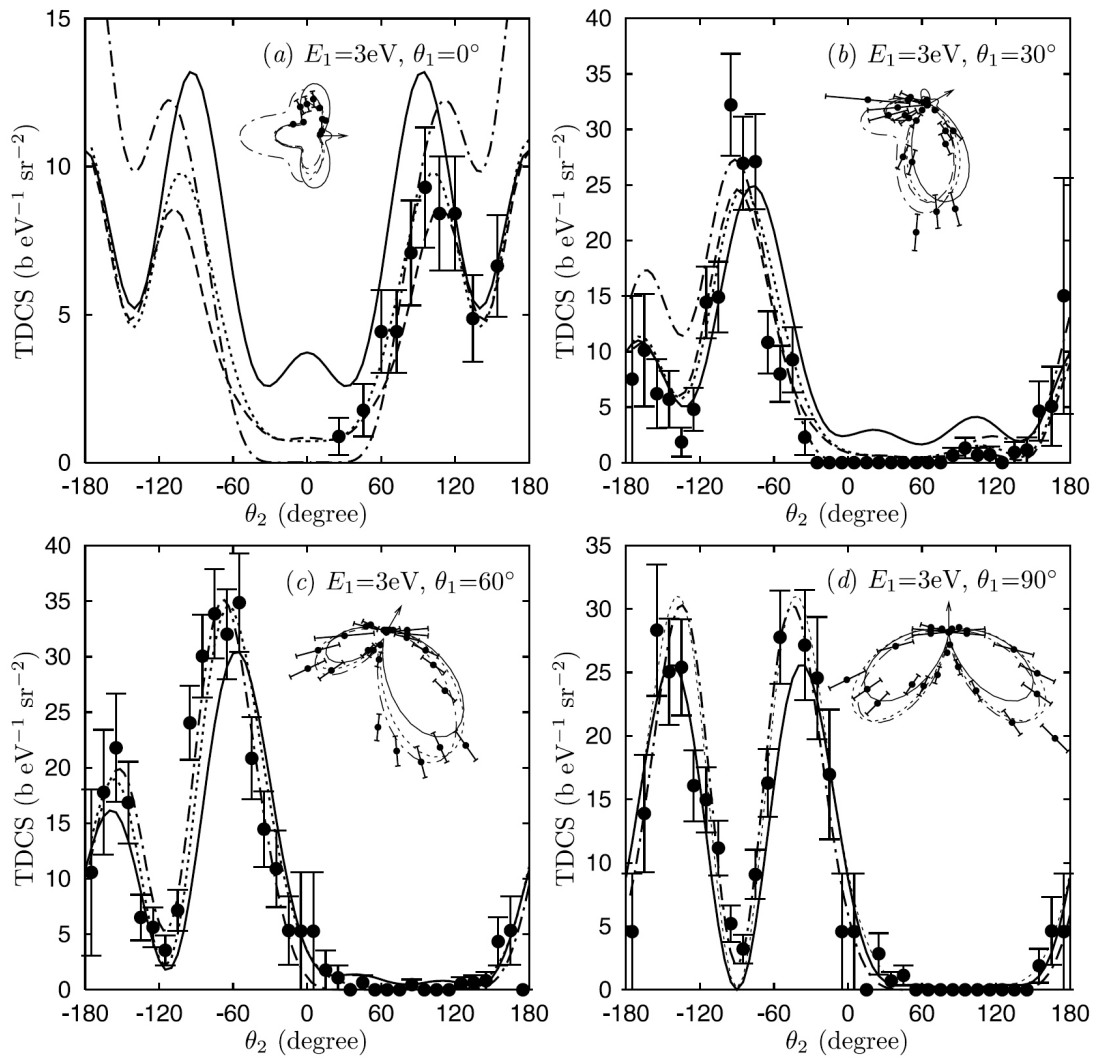

Figure 5. The same as Figure 4, but for unequal energy sharing, $E_{1}=3 \mathrm{eV}$ and $E_{2}=17 \mathrm{eV}$. (Results of Kheifets and Bray are from $[9,13]$.

cal outgoing waves (HRM-SOW) results of Selles et al. [14] and with the time-dependent close-coupling (TDCC) results of Colgan et al. [15]*. One sees that our perturbative predictions for the TDCS are in excellent agreement overall with both the absolute experimental data and the non-perturbative theoretical results. We find similarly good overall agreement of our results [19] with absolute experimental data for the various other energy-sharing cases in [9]. In most cases, the major discrepancies occur over a range of angles centered about $\theta_{12}=0^{\circ}$, which is a direct manifestation of the inadequacy of a firstorder PT account of the strong Coulomb repulsion between the two outgoing electrons when they are ejected at relatively small mutual angles. For equal energy sharing (Figure 4), for $\theta_{1}=0^{\circ}$ the major discrepancies occur for $-40^{\circ} \leq \theta_{2} \leq+40^{\circ}$; for $\theta_{1}=30^{\circ}$ the discrepancies are in the range $0^{\circ} \leq \theta_{2} \leq 60^{\circ}$; for $\theta_{1}=60^{\circ}$ and $90^{\circ}$ the predictions lie within the experimental error bars. For unequal energy sharing, when $E_{1}$ is small and $\theta_{1}$ is also small, as in Figures 5(a) and (b) for $\theta_{1}=0^{\circ}$ and $30^{\circ}$, the discrepancies appear to extend over a wider range than when $E_{1}$ is large and $\theta_{1}$ is small, as in Figures 6(a) and (b). As in the case of equal energy sharing, for $\theta_{1}=60^{\circ}$ and $90^{\circ}$ the predictions generally lie within or close to the experimental error bars (the main exception being around $\theta_{2}=180^{\circ}$ in Figure $6(\mathrm{~d}))$. We note finally that although the energy sharing shown in Figures 5 and 6 may seem quite asymmetric, it is still far from the energy-sharing kinematics at which the SO process dominates [16].

* Note that the experimental data presented in Figure 6 of [15] have been inadvertently transposed, i.e. the measured data for $R=E_{1} / E_{2}$ were presented in the plots corresponding to $E_{2} / E_{1}[15]$. 

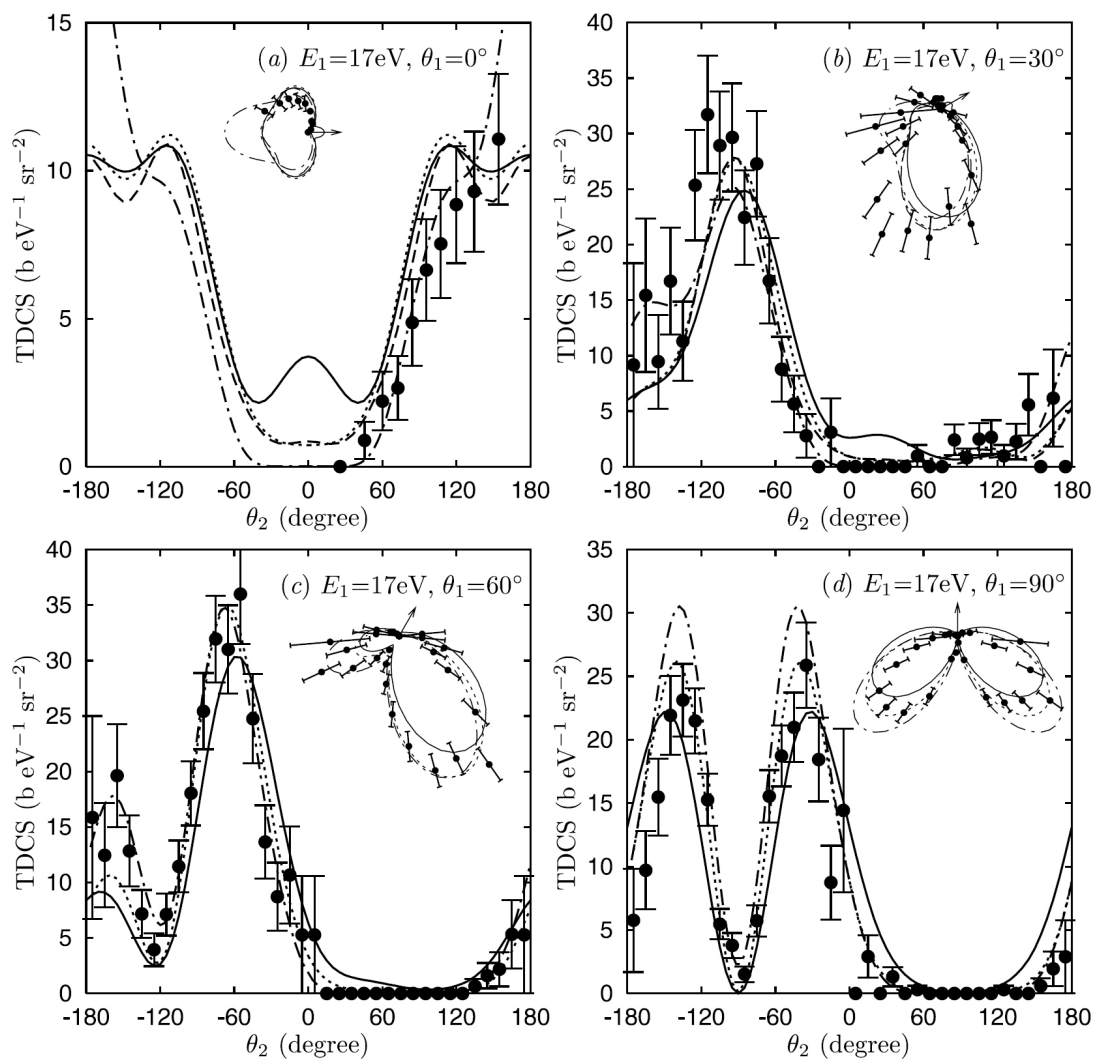

Figure 6. The same as Figure 5, but for $E_{1}=17 \mathrm{eV}$ and $E_{2}=3 \mathrm{eV}$.

In summary, we have analyzed the use of LOPT to describe the TDCS for PDI of He at an excess energy of $20 \mathrm{eV}$, for which absolute data are available [9]. First, we have examined two versions of LOPT using $Z=2$ Coulomb functions and found that both versions fail to describe the experimental data owing to the large GSC amplitudes. Also, one of these versions, intended to reproduce results of [11], fails to do so; specifically, both our FSC and GSC amplitudes give very different results from those of [11]. In a second set of calculations, we have shown that account of FSC to first order using a basis set of $Z=27 / 16$ Coulomb functions provides accurate results for nearly all kinematics of the process, except in the vicinity of $\theta_{12}=0^{\circ}$. This allows us to conclude that the TS- 1 is the dominant mechanism of PDI for the kinematical situations considered. Finally, by comparing our exact treatment of the LOPT TDCS with results of a partial wave analysis, we have shown that only s, p, and d waves contribute significantly to the TDCS over a broad range of mutual angles. This fact allows for parameterization of the PDI amplitude by only a few complex numbers [19]. The contributions of $f$ and higher partial waves are mainly significant at small mutual angles, particularly for $\theta_{1}=\theta_{2}=0^{\circ}$. Details of our method will be presented elsewhere [19] together with an analysis of light polarization effects in the PDI process.

\section{Acknowledgments}

We wish to thank H. Bräuning, I. Bray, J. Colgan, R. Dörner, L. Malegat, and M. Pindzola for providing their data to us and S. Keller for helpful correspondence. This work has been supported in part by the U.S. Department of Energy, Office of Science, Division of 
Chemical Sciences under grant no DE-FG03-96ER14646 and by the Russian Foundation for Basic Research under grant no 03-02-16268. We acknowledge also use of the Research Computing Facility of the University of Nebraska-Lincoln.

\section{References}

[1] Briggs J F and Schmidt V 2000 J. Phys. B: At. Mol. Opt. Phys. 33 R1

[2] King G C and Avaldi L 2000 J. Phys. B: At. Mol. Opt. Phys. 33 R215

[3] Byron F W and Joachain C J 1967 Phys. Rev. 1641

[4] Amusia M Y, Drukarev E G, Gorshkov V G, and Kazachkov M P 1975 J. Phys. B: At. Mol. Phys. 81248

[5] Carter S L and Kelly H P 1981 Phys. Rev. A 24170

[6] Ishihara T, Hino K, and McGuire J H 1991 Phys. Rev. A 44 R6980

[7] Hino K, Ishihara T, Shimizu F, Toshima N, and McGuire J H 1993 Phys. Rev. A 481271

[8] Pan C and Kelly H P 1995 J. Phys. B: At. Mol. Opt. Phys. 285001

[9] Bräuning H et al 1998 J. Phys. B: At. Mol. Opt. Phys. 315149

[10] Pont M, Shakeshaft R, Maulbetsch F, and Briggs J S 1996 Phys. Rev. A 533671

[11] Keller S 2000 J. Phys. B: At. Mol. Opt. Phys. 33 L513 ; Keller S 2002 Private communication

[12] Kheifets A S and Bray I 1998 J. Phys. B: At. Mol. Opt. Phys. 31 L447

[13] Kheifets A S and Bray I 1998 Phys. Rev. Lett. 814588

[14] Malegat L, Selles P, and Kazansky A K 2000 Phys. Rev. Lett. 85 4450; Selles P, Malegat L and Kazansky A K 2002 Phys. Rev. A 65032711

[15] Colgan J, Pindzola M S, and Robicheaux F 2001 J. Phys. B: At. Mol. Opt. Phys. 34 L457; Colgan J and Pindzola M S 2002 Phys. Rev. A 65 032729; Colgan J 2002 Private communication

[16] Knapp A et al 2002 Phys. Rev. Lett. 89033004

[17] Dalgarno A and Sadeghpour H R 1992 Phys. Rev. A 46 R3591

[18] Lucey S P, Rasch J, Whelan C T, and Walters H R J 1998 J. Phys. B: At. Mol. Opt. Phys. 31 1237

[19] Istomin A Y, Manakov N L, and Starace A F, in preparation

[20] Manakov N L and Rapoport L P 1972 Opt. Spectrosk. 33998 (Engl. transl. 1972 Opt. Spectrosc. 33 547); Manakov N L, Marmo S I, and Fainshtein A G 1995 Zh. Exp. Teor. Fiz. 108 1569 (Engl. transl. 1995 Sov. Phys.-JETP 81 860)

[21] Schneider T, Chocian P L, and Rost J M 2002 Phys. Rev. Lett. 89073002 